\title{
DC Motor Speed Control Based on Endocrine Regulation Mechanism
}

\author{
Hua Meng \\ Hebei University of Science and Technology \\ Department of Electrical Engineering \\ Shijiazhuang, China \\ menghua0311@126.com \\ Boyang Zhao* \\ Hebei University of Science and Technology \\ Department of Electrical Engineering \\ Shijiazhuang, China \\ laobaiup@126.com
}

\author{
Hui Gao \\ Hebei University of Science and Technology \\ Department of Electrical Engineering \\ Shijiazhuang, China \\ gaohuigogh@126.com \\ Xiaohong Zheng \\ Hebei University of Science and Technology \\ Department of Electrical Engineering \\ Shijiazhuang, China \\ 15614120310@163.com
}

\begin{abstract}
The DC motor of Control system is a control algorithm for most PID. In this paper, a kind of intelligent ultra short feedback endocrine controller is designed, which is based on the mechanism of hormone secretion regulation, and is applied to the control of DC motor. The endocrine controller includes the traditional control unit, the ultra short feedback unit and so on. First the control signal of the endocrine controller is operated by the traditional control unit. Then the control signal is processed by the ultra short feedback unit according to the regulation of hormone. In the end, the output signal of the ultra short feedback unit and the output signal of the traditional control unit are combined and output, thus a nonlinear control algorithm is formed. The new control algorithm is applied to the DC servo motor model and the three - level water tank model. This paper conducts a simulation comparison through programming. The simulation results show that the controller has good stability, fast performance and anti disturbance ability with the traditional PID control.
\end{abstract}

Keywords- DC Motor; Intelligent Controller; Neuroendocrine; Ultra Short Feedback; Hormone

\section{INTRODUCTION}

With the development of modern society in the development of human industry, modern industrial control requirements are more complex, the traditional control theory and technology can not meet the requirements of modern industry. Therefore, the development of new control theory and technology is very important. Other areas of science will have a great reference to the development of control theory, so the intelligent control system based on biological network came into being.

Intelligent control system based on biological network, which can also be called artificial intelligence system. This information processing system can be divided into brain nerve system, genetic system, immune system, endocrine system and so on. Artificial neural network (ANN), genetic algorithm (GA), artificial immune system (AIS), artificial endocrine system (AES), and artificial immune system (EC) are proposed. The study of artificial endocrine system started late and the research results were the least, that is, the potential of its development is enormous.

In many of the mechanisms of biological information processing, endocrine regulation mechanism is a very important part, and the biological behavior is influenced by the regulation of the endocrine system, such as regulation of thyroid hormone, testosterone regulation etc. Artificial endocrine system (AES) is now the main content of the study is modeled on the endocrine regulation mechanism (hormone regulation) to complete the design of the controller of hormone regulation mechanism.

From the beginning of the 1970s, biological control theory had gradually attracted the attention of researchers and developed rapidly [1]. In the 1980s, the neural network research boomed up and made great achievements. At present, neural network control has developed in a more mature manner[2]. However, there are less reports and researches about the biological intelligence control theory based on neuroendocrine system, and the study of the endocrine system had just started. Although some scholars put forward several kinds of hormone regulation simulation model, the model or the modeling theory is mainly applied in the field of medical research. As the control center of various human hormones, neuroendocrine system can regulate a variety of hormones, with better adaptability and stability. Therefore, the research on the biological intelligent control theory based on the neuroendocrine system will have an impact on the development of the traditional control theory, which can improve the control quality of the complex object[3].

Endocrine regulation system, artificial neural networks and artificial immune systems are collectively known as the three regulatory mechanisms of the body. There are a lot of researches and achievements about neural network, but the research about immune system is at the starting stage[5].While some scholars have proposed several hormonal regulation simulation models, these models and 
modeling theories are mainly used in medical research. In this paper, a kind of intelligent ultra short feedback endocrine controller is designed, which is based on the mechanism of hormone secretion regulation, and is applied to the control of DC motor. The endocrine controller includes the traditional control unit, the ultra short feedback unit and so on. First the control signal of the endocrine controller is operated by the traditional control unit[4]. Then the control signal is processed by the ultra short feedback unit according to the regulation of hormone. In the end, the output signal of the ultra short feedback unit and the output signal of the traditional control unit are combined and output, thus a nonlinear control algorithm is formed. By reasonably designing the operation rule of the ultra short feedback unit, the suppression or enhancement of control output is realized, and the control effect is improved.

\section{DESIGN OF ENDOCRINE SHORT FEEDBACK CONTROLLER}

Endocrine regulation system, artificial neural networks and artificial immune systems are collectively known as the three regulatory mechanisms of the body. There are a lot of researches and achievements about neural network, but the research about immune system is at the starting point[5].While some scholars have proposed several hormonal regulation simulation models, but these models and modeling theories are mainly used in medical research. The ability of endocrine system to regulate various hormones has good adaptability stability. Hormones are substances which have an active chemical character and are secreted by endocrine glands or dispersed endocrine cells. The active substances use various liquid in the body as medium, transfer chemical information between the cells. The organs, tissues and cells which are regulated by hormones are called target organ, target tissue, and target cells[6]. There are several ways for hormones to regulate the body's life activities, such as telecrine, paracrine, autocrine and neurosecretion. Most hormones is secreted by endocrine cells at the beginning, and then transported to the target tissue, target cells through the blood circulation and play its due role, and this kind of method is called telecrine; Some hormones which are secrete by endocrine cells enter into the tissue fluid nearby, and then spread to the proximity target cells, and this kind of method is called paracrine;

There are also some hormones that will affect its original cells after secreted to regulate the original cells themselves and other similar cells nearby, this kind of approach is called autocrine; By delivery through a variety of ways, one hormone can play a lot of roles simultaneously[7].

The function of the regulation, control and feedback of endocrine system is achieved by hormone regulation mainly. Hormone regulation allows the body to function more completely. There are many hormones to regulate the endocrine system. And there are multi-levels of regulation between the different hormones. Under the action of the central nervous system and hormone feedback regulation, the organism adaptation to the environment tends to be more perfect. As shown in Fig. 1, the regulatory function of the endocrine system is mainly reflected in two aspects: one is the hypothalamus - pituitary - target gland system, which plays an irreplaceable role in endocrine regulation process [8]. In general, the hypothalamic regulated by the central nervous system releases hormones which stimulate the pituitary endocrine cell to release trophic hormone, and the trophic hormone will act on periphery glands and promote their activities; endocrine cells on the periphery glands secrete hormones and the hormones will have a feedback regulation on the activity of the gland. And these feedback regulations mostly are negative feedback effect. Thus by the mutual adjustment of the endocrine glands, the internal environment can maintain in a balance situation. On the other hand, it refers to the independent endocrine glands which can release the hormones to stimulate the target tissue to generate the corresponding effects. As shown in Fig. 1:

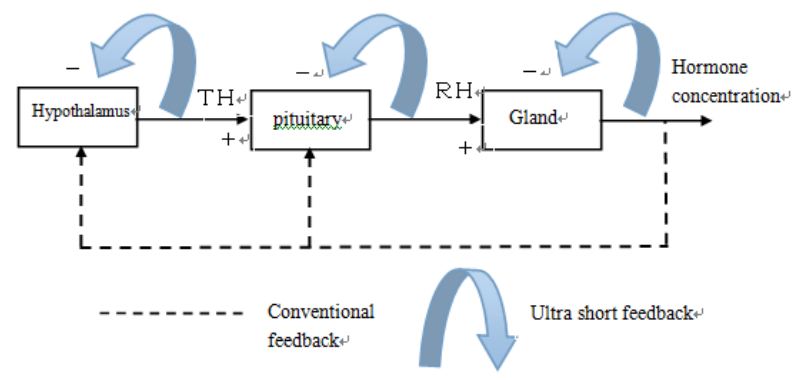

Figure 1. Endocrine ultrashort feedback loop

Inspired by the hormonal regulation of ultrashort feedback, this paper designs an ultrashort feedback unit. As shown in Fig. 2, by reasonably designing the operation rule of the ultra short feedback unit, the control output is suppressed or enhanced, which can improve control effect.

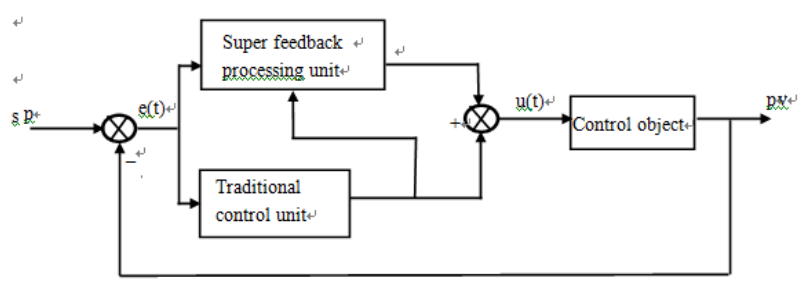

Figure 2. Ultra short feedback controller structure diagram

Researchers found the general rule of the hormone gland secretion through the research: The change of hormones is monotonous and non negative, the rise and fall of hormone secretion regulation follows the rule of Hill function, Equation (2.1) and Equation (2.2) are shown.

$$
\begin{aligned}
& F_{\text {up }}(G)=\frac{G^{n}}{T^{n}+G^{n}} \\
& F_{\text {down }}(G)=\frac{T^{n}}{T^{n}+G^{n}}
\end{aligned}
$$

Where $\mathrm{G}$ is a function of the independent variable; $\mathrm{T}>0$, and $\mathrm{t}$ is the threshold; $\mathrm{n}$ is more than or equal to 1 , the Hill coefficient; $\mathrm{N}$ and $\mathrm{t}$ control curve rising and falling slope. The function has the following properties:

$$
\begin{aligned}
& \text { (1) } F_{u p}=1-F_{d o w n} \\
& \text { (2) } F_{u p,(d o w n)}(T)=1 / 2 \\
& \text { (3) } 0 \leq F_{u p,(\text { down })}(G) \leq 1
\end{aligned}
$$


If the hormone $\mathrm{x}$ is controlled by the hormone $\mathrm{x}$, the secretion rate of the hormone $y$ is shown to be related to the concentration of the hormone y (2.3) :

$$
S_{x}=a F_{\text {up },(\text { down })}\left(C_{y}\right)+S_{x, \text { basal }}
$$

Among them, $\mathrm{Cy}$ is the concentration of the hormone $\mathrm{y}$, $\mathrm{S}$, basal is the base of hormone $\mathrm{x}$ secretion rate, $\mathrm{a}$ is the constant coefficient[9].

In the Fig. 2, the output signal uc(t) of the controller structure in traditional control unit, the first feedback to ultrashort feedback processing unit and then ultrashort feedback processing unit in accordance with the nonlinear function $\mathrm{f}(\mathrm{u}(\mathrm{t}))$ treat it, the processed signal is superimposed with the traditional control unit output signal, which can improve the control effect by inhibiting or enhancing the control output. The control law of hormone regulation is made by designing the nonlinear processing function of the ultra short feedback. The change rate of the control output signal $\triangle \mathrm{uc}(\mathrm{t})$ is used as the regulation of the hormone regulation, according to the regulation of hormone regulation, the nonlinear processing function of the output signal is obtained by (2.4) (2.5) (2.6):

$$
\begin{gathered}
f\left(\square u_{c}(k)\right)=a *\left(\frac{\left(\left.\square u_{c}(k)\right|^{n}\right)}{1+\left.\square u_{c}(k)\right|^{n}}+b\right) L_{1} * L_{2} \\
L_{1}=-\frac{e(k)}{|e(k)|} * \frac{\square e(k)}{\square e(k) \mid} \\
L_{2}=\frac{\square u(k)}{\square u(k) \mid}
\end{gathered}
$$

Where $\triangle \mathrm{uc}(\mathrm{k})=\mathrm{uc}(\mathrm{k})-\mathrm{uc}(\mathrm{k}-1), \mathrm{k}$ is the number of steps, a, b, $\mathrm{n}$ are all factor coefficient. And the value at least when $\triangle \mathrm{uc}(\mathrm{k}), \mathrm{f}(\mathrm{uc}(\mathrm{k}))=0$. That b is $0 . \mathrm{L} 1$ represents the positive and negative product of the positive and negative product of the positive and negative product of the positive and negative value of the control deviation; L2 represents the change direction of the output. L1 and L2 guarantee that the control effect is enhanced when the process variables change in the direction of the setting value; on the contrary, the control effect is weakened. Then the incremental output of the controller is shown in (2.7):

$$
\square u(k)=\square u_{c}(k)+a *\left(\frac{\left(\left.\square u_{c}(k)\right|^{n}\right)}{1+\left.\square u_{c}(k)\right|^{n}}\right) L_{1} * L 2
$$

Where $\triangle \mathrm{uc}(\mathrm{k})$ is the output change of traditional control unit. The algorithm of the traditional PID controller is shown in the formula (2.8):

$$
\square u_{c}(k)=K_{p}\left\{\begin{array}{l}
{[e(k)-e(k-1)]+T_{s} \frac{e(k)}{T_{i}}+} \\
T_{d} \frac{[e(k)-2 e(k-1)+e(k-2)]}{T_{s}}
\end{array}\right\}
$$

The output expression of the controller is shown in (2.9):

$$
\begin{aligned}
& \square u(k+1)=K_{p}\left\{\begin{array}{l}
{[e(k)-e(k-1)]+T_{s} \frac{e(k)}{T_{i}}+} \\
T_{d} \frac{[e(k)-2 e(k-1)+e(k-2)]}{T_{s}}
\end{array}\right\} \\
& +a *\left(\frac{\left(\left.\square u_{c}(k)\right|^{n}\right)}{1+\left.\square u_{c}(k)\right|^{n}}\right) L_{1} * L 2
\end{aligned}
$$

\section{SIMULATION VERIFICATION}

DC servo motor, also known as the motor, from the control motor, is commonly used in the control system components. DC servo motor is able to shift the input voltage signal into angular displacement and angular velocity signal output. Servo system is a common system in control system[10]. Servo system composed of servo motor can be divided into position mode, speed mode and torque control mode. Among them, the first two are the most common, in the control system.

DC servo motor has the following characteristics:

It is convenient to realize stepless speed regulation in a wide range, so it is used in the situation of high speed performance for the motor.

Other DC motor armature control is similar to the corresponding characteristics; DC servo motor has the advantages of high starting torque, small starting current, wide adjustment range, and so on.

The output power of the servo motor is higher than the other motor.

Servo motor speed regulation has a very high accuracy, speed range wide, the capability of driving load is strong, the braking performance and the dynamic performance are good.

Because of the smaller rotary inertia of the rotor, the servo motor is superior in quality and volume than the other motor with the same power.

Compared with other complicated structures, the advantages of the servo motor are high reliability, good stability, wide working range, simple maintenance and maintenance.

High efficiency, its efficiency is higher than the other asynchronous motor with same capacity[11].

Motor speed regulation is realized by changing the armature voltage. Changing the armature voltage is mainly to change the PWM (pulse width modulation converter). PWM (pulse width modulation) converter is also known as DC chopper. Its working principle is: Through the power switch device, which achieves to adjust the ratio of time, in this way, the fixed DC voltage can be turned into an adjustable DC voltage. Therefore PWM (pulse width modulation) converter is also known as the DC - DC converter. Therefore, the speed control of the motor can be realized by controlling the signal of PWM.

In order to verify the control performance of the ultra short feedback controller in the motor control, we choose a DC motor. DC motor's parameters are as follows:

Rated voltage: $\mathrm{UN}=24 \mathrm{~V}$;

Rated current: $\mathrm{IN}=5 \mathrm{~A}$;

Rated powe: $\mathrm{PN}=80 \mathrm{~W}$;

Rated speed: $\mathrm{nN}=1500 \mathrm{n} / \mathrm{min}$;

Torque coefficient: $\mathrm{Cm}=0.01 \mathrm{~N} * \mathrm{~m} / \mathrm{A}$; 
Induced electromotive force coefficient: $\mathrm{Ce}=0.1 \mathrm{~N} * \mathrm{~m} / \mathrm{A}$;

Total resistance of armature loop: $\mathrm{R}=1.6 \Omega$;

Total inductance of armature loop: $\mathrm{L}=2.6 \mathrm{mH}$;

Motor and load torque of motor shaft:

$U_{\mathrm{d} 0}(s)=R I_{\mathrm{d}}(s)+L s I_{\mathrm{d}}(s)+E_{\mathrm{d}}(s)$

Electromechanical time constant:

$T_{m}=\frac{D G^{2} R}{375 C_{m} C_{e}}=0.048 \mathrm{~s}$

Electromagnetic time constant:

$T_{l}=\frac{L}{R}=0.016 s$

The main circuit current is continuous, motor dynamic voltage equation:

$$
U_{\mathrm{d} 0}=R I_{\mathrm{d}}+L \frac{\mathrm{d} I_{\mathrm{d}}}{\mathrm{d} t}+E_{d}
$$

Under zero initial conditions, the pull type transformation is obtained:

$U_{\mathrm{d} 0}(s)=R I_{\mathrm{d}}(s)+L s I_{\mathrm{d}}(s)+E_{\mathrm{d}}(s)$

Get:

$$
\frac{I_{\mathrm{d}}(s)}{U_{\mathrm{d} 0}(s)-E_{\mathrm{d}}(s)}=\frac{1 / R}{1+T_{l} s}
$$

Therefore, the transfer function of the controlled object motor is (3.1):

$$
G(s)=\frac{400}{s(s+50)}
$$

PID control's parameters are is: $\mathrm{kp}=10, \mathrm{ki}=0.01, \mathrm{kd}=3$. ltra short feedback parameters is: $\mathrm{a}=1.8, \mathrm{~b}=0, \mathrm{n}=1$.2. Simulation results are shown in Figure . Fig. 3. The response of the motor for the traditional PID control to the step signal is shown in Fig. 4. The response of effect diagram of the electric machine with the ultra short feedback control for step signal is also shown.

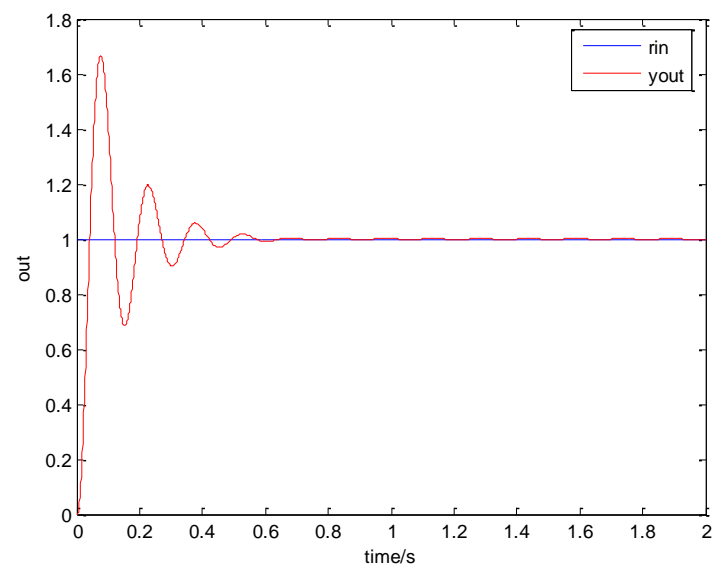

Figure 3. The motor for the traditional PID

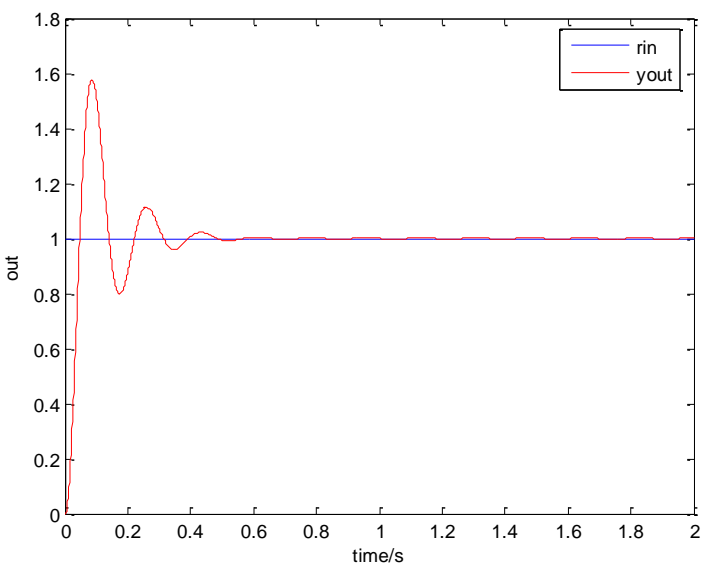

Figure 4. The motor with the ultra short feedback control

By observing two simulation results, we found that Join the ultrashort feedback unit control algorithm in nearly $0.5 \mathrm{~s}$ basically meet the requirement of control, but the traditional PID control algorithm is nearly $0.6 \mathrm{~s}$, control effect for nearly $0.1 \mathrm{~s}$ ahead of time, and overshoot volume decreased significantly. The simulation results show that relative to the traditional PID control, the ultra-short feedback unit controller has better stability and rapidity.

In order to further verify the application of the ultra short feedback controller in practice. In this paper, a three stage liquid level control object is selected as the object of simulation the transfer function is (3.2):

$$
G(s)=\frac{2}{s^{3}+3 s^{2}+3 s+1}
$$

In order to facilitate the comparison of the control effect, the PID control parameters of the ultra short feedback controller and the control parameters of the conventional PID controller are the same. ID control's parameters are: $\mathrm{kp}=0.05, \mathrm{ki}=0.027, \mathrm{kd}=0$. Ultra short feedback parameters is: $a=2, b=0, n=1$.3. Simulation results are shown in Fig. 5. The response of the liquid level control object for the traditional PID control to the step signal is shown in Fig. 6. The response of effect diagram of the liquid level control object with the ultra short feedback control for step signal is also shown.

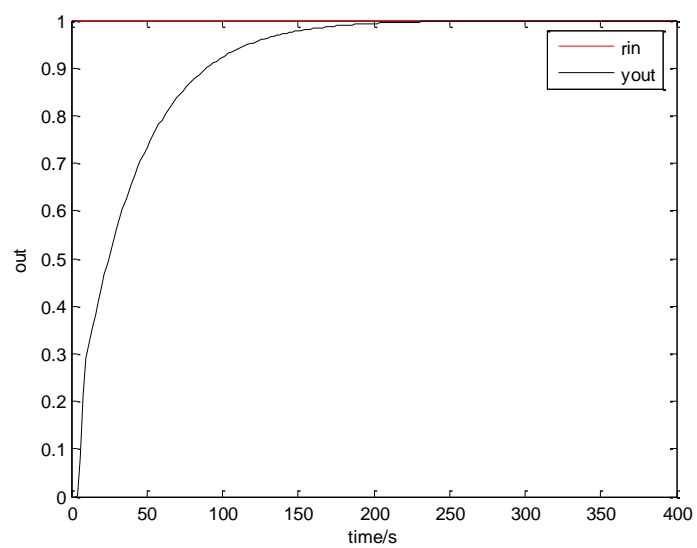

Figure 5. The liquid level control object for the traditional PID 


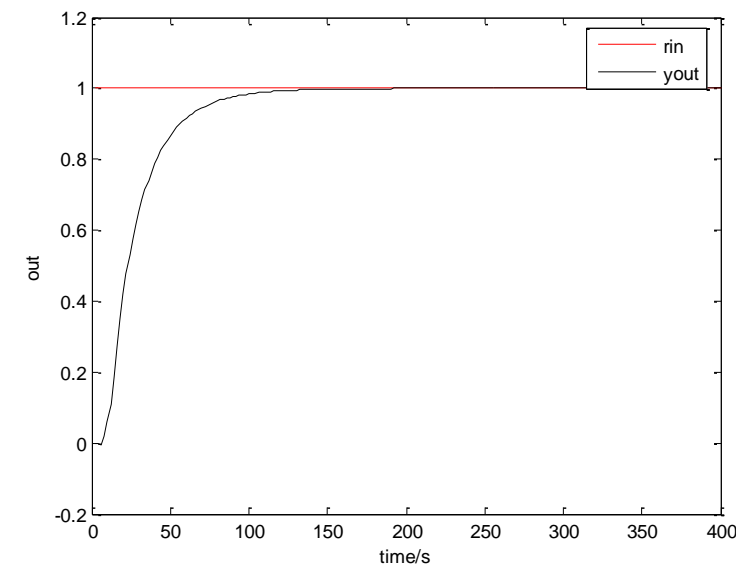

Figure 6. The liquid level control object with the ultra short feedback control

By observing two simulation results, we found that Join the ultrashort feedback unit control algorithm in nearly 120 s basically meet the requirement of control, but the traditional PID control algorithm is nearly 205s, control effect for nearly $85 \mathrm{~s}$ ahead of time, and overshoot volume decreased significantly. The simulation results show that, relative to the traditional PID control, join the ultra-short feedback unit controller has better stability and rapidity.

\section{CONCLUSIONS}

In this paper, a kind of intelligent ultra short feedback endocrine controller is designed, which is based on the mechanism of hormone secretion regulation, and is applied to the control of DC motor. The endocrine controller includes the traditional control unit, the ultra short feedback unit and so on. First the control signal of the endocrine controller is operated by the traditional control unit. Then the control signal is processed by the ultra short feedback unit according to the regulation of hormone. In the end, the output signal of the ultra short feedback unit and the output signal of the traditional control unit are combined and output, thus a nonlinear control algorithm is formed. The new control algorithm is applied to the DC servo motor model and the three - level water tank model. This paper conducts the simulation comparison through programming. The simulation results show that the controller has good stability, fast performance and anti disturbance ability with the traditional PID control.

\section{REFERENCES}

[1] Zhang D, Gao Z, "Optimal kinematic calibration of parallel manipulators with pseudo error theory and xooperative coevlutionaty netword [J]"IEEE Transactions on Industrial Electronics,2012,59(8):3221-3231.

[2] Zhang D, Lei J. Kinematic analysis of a novel 3-DOF actuation redundant parallel manipulator using artificial intelligence approach $[\mathrm{J}]$. Rpbotics and Computer-Integrated Manufactring,2011,27(1):157 163.

[3] Khoujhi A.Data-driven multi-stage motion planning of paralle kinematic machines[J].IEEE Transactions on Control Systems Technong,2010,18(6):1381_1389.

[4] Cheng C-H ,Shu S-L.Application of GA-based neural network for attitude control of a satellite [J].Aerospace Science and Technology,2010,14 (4):241_249.

[5] Chen R X, Ren L H, Ding Y S.neuroendocrine-based intelligent control of a 6-DOF parallel robot with redundant drivers. The 2008 Chinese and Decision Conference(2008 CCDC), Yantai,China,2008:2068 2071.

[6] Timmis J, Neal M. Artificial homeostasis:Integrating biologically inspiredcomputing,www.cs.kent.ac.uk/2003/1586/content.

[7] Kravitz E A.Hormonal control for behavior:Amines and the biasing of output in lobsters. Science, 1998,241:1775 178.

[8] Tian Jiangjun, Application of DSP controlled brushless DC motor in transfusion pump . Modern Machinery:2013

[9] Maher Mahmoud Abdo, Ahmad Reza Vali, Ali Reza Toloei, Mohammad Reza ArvanStabilization loop of a two axes gimbal system using self-tuning PID type fuzzy controllerISA Trans., 53 (2014), pp. 591-602

[10] Y.Sh. Kung, R.F. Fung, T.Y. Tai Realization of a motion control IC for $\mathrm{X}-\mathrm{Y}$ table based on novel FPGA technology IEEE Trans Industr Electron, 56 (1) (2009), pp. 43-53.

[11] B.M. Hamed, M.S. El-MoghanyFuzzy controller design using FPGA for sun tracking in solar array systemI.J. Intell Syst Appl, 1 (February) (2012), pp. 46-52 . 